\title{
An Untraceable Off-Line Electronic Cash Scheme without Merchant Frauds
}

\author{
Baoyuan Kang and Danhui Xu \\ School of Computer science and software \\ Tianjin polytechnic university, Tianjin, 300387, China \\ baoyuankang@aliyun.com,mixueren123123@sina.com
}

\begin{abstract}
In an electronic cash scheme, there are three participants, the bank, the spender and the merchant. First, a spender opens an account in a bank. Then, he withdraws electronic cash from his account and pays it to a merchant. After checking the electronic cash's validity, the merchant accepts it and deposits it to the bank. There are a number of requirements for an electronic cash scheme, such as, anonymity, unforgeability, unreusability, date attachability, divisibility, transferability and portability. In this paper, we show a recently proposed electronic cash scheme is suffering from some faults in anonymity, expiration date and merchant frauds. To improve the scheme, we propose a new untraceable off-line electronic cash scheme and give a security analysis for it.
\end{abstract}

Keywords: Electronic cash, Payment system, Anonymity, Signature scheme, Cryptography

\section{Introduction}

Due to the fast progress of computer networks and Internet, information technology is used in electronic commerce. Electronic payment is one of the key issues of electronic commerce development. To realize the digitalization of traditional cash, in 1983, Chaum suggested the first electronic cash scheme [1]. Popularly, in an electronic cash scheme, there are three participants, the bank, the spender and the merchant. First, a spender opens an account in a bank. Then, he withdraws electronic cash from his account and pays it to a merchant. After checking the electronic cash's validity, the merchant accepts it and deposits it to the bank. For security and efficiency, there are a number of requirements for electronic cash scheme, such as, anonymity, unforgeability, unreusability, date attachability, divisibility, transferability and portability [2]. Some of them are listed below:

Anonymity: The spender of a cash must be anonymous. As long as the coin is spent legitimately, neither the merchant nor the bank can identify the spender of the coin.

Unforgeability: Only authorized banks can generate electronic cash.

Unreusability: The electronic cash cannot be reused. The scheme can detect the

malicious spenders, who spend the cash twice.

Date attachability: Electronic cash must embody the dates of withdrawing, paying and depositing. These dates can are used to check the expiration date and charge for interest.

Electronic cash schemes can be divided into two categories: online and off-line. In online schemes, as paying a coin to a merchant, the bank must attend to validate the coin and detect its reuse. But, in off-line schemes, double spending can only be figured out when the merchant deposits the coin to the bank in the next phase. After Chaum's scheme, a lot of electronic cash schemes [3-8] have been proposed based on blind signature and restrictive blind signature [9]. Afterward, many more complex schemes have been proposed [10-13]. Recently, Eslami and Talebi proposed an untraceable electronic cash scheme [2] and claimed that their scheme satisfies all main security 
requirements, such as, anonymity, unreusability and date attachability. However, Baseri, et al., [14] showed that Eslami and Talebi's scheme is subjected to some weaknesses in perceptibility of double spender, unforgeability and date attachability. Baseri, et al., also contributed an electronic cash scheme and claimed that their scheme is immune to the weaknesses of Eslami and Talebi's scheme. But, as we show in this paper, Baseri, et al.,'s scheme is suffering from some faults in anonymity, expiration date and merchant frauds. To improve Baseri, et al.,'s scheme, we also propose a new untraceable off-line electronic cash scheme. The new scheme not only possesses the features, such as anonymity, unforgeability, unreusability, but also possesses the feature of avoiding merchant frauds.

The remainder of this paper is organized as follows. Some basic concepts are introduced in Section 2. In Section 3, we review Baseri, et al.,'s scheme and show its weaknesses. In Section 4 we propose a new electronic cash scheme. Security analysis of our scheme is covered in Section 5. Performance comparisons are shown in Section 6. We finally conclude in Section 7.

\section{Preliminaries}

\subsection{RSA Cryptosystem}

A RSA cryptosystem [15] can be determined by a set $(p, q, n, e, d)$. Where $p$ and $q$ are two large prime numbers, $n=p q$ and $e d=1(\bmod \phi(n))$. Here $\phi(n)=(p-1)(q-1) .(p, q, d)$ are private while $(n, e)$ are public. For encrypting the plain text $m$, one calculates the cipher $c$ as $c=m^{e}(\bmod n)$. For decrypting $c$, one calculates the plain text $m$ as $m=c^{d}(\bmod n)$.

\subsection{Blind Signatures}

In 1983, Chaum proposed a blind signature scheme [1] based on the RSA cryptosystem. Blind signatures can be applied to preserve the anonymity of users against leaking user information to the signer, such as, in electronic cash payment and electronic voting schemes. A typical blind signature scheme involves two participants: a signer and a signature requester. The signature requester need the signature of the signer on one message $m$. But the requester does not wish to leak the content of $m$ to the signer. Hence, the requester chooses a random number $b$ as a blinding factor and sends $b^{e} m$ ( $e$ is the signer's public key in RSA cryptosystem) to the signer. The signer computes $\left(b^{e} m\right)^{d}$ ( $d$ is the signer's private key related to public key $e)$. Since $\left(b^{e} m\right)^{d}=b m^{d}$, the requester can obtain the signature $m^{d}$ on $m$ by multiplying $\left(b^{e} m\right)^{d}$ by $b^{-1}$.

\section{Baseri et al.'s Scheme and its Failures}

In this section, we first review Baseri et al.'s scheme [14]. Then we show its failures.

\subsection{Baseri, et al.,'s scheme}

There are four participants in the scheme: a Central Authority, the Bank, the Spender and the Merchant. The scheme contains five phases: initialization, withdrawal, payment, deposit and exchange.

3.1.1 Initialization: In this phase, the central authority should set some public parameters. There parameters include two publicly known elements, $g_{1}, g_{2}$, of the same 
large prime order, $l$ in $Z_{n}^{*}$. Here $n=p q$ and $p, q$ are two large prime numbers. $H$ is a one-way hash function. In addition, each authenticated participant involved in the system should determine his own parameters and get a certificate for its own public key from certification authority. The required parameters of the bank are two RSA public/private key pairs $\left(\left(e_{B}, n\right), 1 / e_{B}\right)$ and $\left(\left(e_{B}{ }^{\prime}, n\right), 1 / e_{B}{ }^{\prime}\right)$ such that $e_{B}>e_{B}{ }^{\prime}$.

3.1.2 Opening an Account: To open an account, the customer should identify himself to the bank. Authenticating the customer, the bank stores his identity information in its account database. This process is done in the following steps.

Step 1. The customer:

(a) Identifies himself by means of official documents, like a passport or some other identification.

(b) Generates a random number, $u \in_{R} Z_{n}^{*}$, and keeps it as his own secret identity information which is unknown to any other, unless he spends a coin more than one time.

(c) Computes:

$I D_{c}=g_{1}^{u}(\bmod n)$ as his identity, such that $g_{1}^{u} g_{2} \neq 1(\bmod n)$.

(d) Send $I D_{c}$ to the bank.

(e) Provides a zero knowledge proof that he knows the discrete logarithm of $I D_{c}$, which respect to $g_{1}$.

Step 2. The bank $B$ :

(a) Checks the identity and the zero knowledge proof offered by the customer.

(b) Stores the identity information of the customer in the account database.

(c) Computes $A$ and $O_{1}$ as its own signature on $A$ :

$$
\begin{aligned}
& A=I D_{C} g_{2}(\bmod n), \\
& O_{1}=A^{1 / e_{B}}(\bmod n) .
\end{aligned}
$$

(d) Sends $A$ and $O_{1}$ to the customer.

3.1. 3. Withdrawal: Before withdrawing and asking for a coin, the spender should prove his/her ownership of the account to the bank. The spender should prove his identity in a similar way to the withdrawal of classical cash from an account (i.e., by offering his passport or driving license). In addition, he should refer to a bulletin board in which the bank periodically publishes the fresh time by two parameters, $t$ and $e_{B} * t(\bmod \phi(n))$. Time $t$ is constant during the period and used to synchronize customers and the bank in the withdrawing process and to determine the validation time of coins. Note that $t * e_{B}$ plays the role a public key for the bank and is chosen in such a way that its reverse (i.e., $\left.1 /\left(e_{B} * t\right)(\bmod \phi(n))\right)$ exists. The coin is represented by a five-tuple $\left(A^{\prime}, B, s_{1}, s_{2}, s_{3}\right)$ constructed in the following steps

Step 1. The spender S:

(a) Chooses three random numbers, $x_{1}, x_{2} \in{ }_{R} Z_{e_{B}^{\prime}}^{*}$ and $s \in{ }_{R} Z_{n}^{*}$, and two blinding factors $b_{1}, b_{2} \in Z_{n}^{*}$.

(b) Computes:

$$
\begin{aligned}
& \text { - } A^{\prime}=A^{s}(\bmod n), \\
& \text { - } \quad B=g_{1}{ }^{x_{1}} g_{2}{ }^{x_{2}}(\bmod n),
\end{aligned}
$$


- $\omega_{1}=B b_{1}^{e_{B}^{\prime}}(\bmod n)$,

- $\omega_{2}=\left(A^{\prime}+B\right) b_{2}^{\left(e_{B}{ }^{*}\right)}(\bmod n)$.

(c) Sends $\omega_{1}, \omega_{2}, t$ to the bank.

Step 2. The bank B:

(a) Checks the validity of the Date/Time slip.

(b) Signs $\omega_{1}$ and $\omega_{2}$ by computing:

- $O_{2}=\omega_{1}^{1 / e_{B}^{1}}(\bmod n)$,

- $O_{3}=\omega_{2}^{1 /\left(e_{B}{ }^{*} t\right)}(\bmod n)$.

(c) Sends $\mathrm{O}_{2}$ and $\mathrm{O}_{3}$ to the spender.

Step 3. The spender $\mathrm{S}$ :

(a) Verifies the signatures of the bank on $A, \omega_{1}, \omega_{2}$.

(b) Obtains the signatures of the bank on $A^{\prime}, B$ and $A^{\prime}+B$, which are signed with private keys $1 / e_{B}, 1 / e_{B}^{\prime}$ and $\left(1 /\left(e_{B} * t\right)\right)$, respectively:

- $s_{1}=O_{1}^{s}(\bmod n)=\operatorname{sign}_{B}\left(A^{\prime}\right)$,

- $s_{2}=O_{2} / b_{1}(\bmod n)=\operatorname{sign}_{B}(B)$,

- $s_{3}=O_{3} / b_{2}(\bmod n)=\operatorname{sign}_{B}\left(A^{\prime}+B\right)$.

The Coin is $\left(A^{\prime}, B, s_{1}, s_{2}, s_{3}, t\right)$.

3.1.4 Payment: When the spender wants to spend his coin at the shop, the following steps are done:

Step 1. The spender S:

(a) Sends $A^{\prime}, B, s_{1}, s_{2}, s_{3}, t$ to the merchant $\mathrm{M}$.

Step 2. The merchant M:

(a) Verifies if $A^{\prime} \neq 0$.

(b) Checks the expiration date of the coin.

(c) Verifies the signatures, $s_{1}$, using the public key, $e_{B}, s_{2}$ using the public key, $e_{B}^{\prime}$ and $s_{3}$, and using the public key $\left(e_{B}^{*} t\right)$.

(d) Computes:

- The challenge $d=H\left(A^{\prime}, B, I D_{M}\right.$, datell time $)$ in which $H$ is the hash function determined in the initialization phase, $I D_{M}$ is the identity of the merchant and datell time represents the date and time of the transaction.

(e) Sends $d$ to the spender.

Step 3. The spender S:

(a) Computes:

- $r_{1}=d u s+x_{1}\left(\bmod e_{B}\right)$,

- $r_{2}=d s+x_{2}\left(\bmod e_{B}\right)$.

(b) Sends $r_{1}$ and $r_{2}$ to the merchant.

Step 4. The merchant M:

(a) Accepts the coin if $g_{1}^{r_{1}} g_{2}^{r_{2}}=A^{\prime d} B$. 
3.1.5 Deposit: In this phase, the following process is done between the bank B and the merchant M:

Step 1. The merchant M:

Sends the transcript of each electronic coin (i.e. Coin , $r_{1}, r_{2}$ ) to the bank.

Step 2. The bank B:

(a) Checks the authenticity of the merchant and verifies the transcript of the received coin.

(b) Checks whether the coin exists in its deposit or exchange tables or not. If the coin exists, it runs the double spender detection procedure, else, accepts the coin, stores it in the deposit table and transfers money to the merchant.

3.1.5.1. Double Spender Detection Procedure: Suppose that a malicious spender spends the same coin twice or more. Suppose that the malicious spender first spender the coin, along with $d^{\prime}, r_{1}^{\prime}$ and $r_{2}^{\prime}$, the bank finds out that the coin already exists in its tables. At that time, using the relation between $r_{1}, r_{2}, d$ and consequently between $r_{1}^{\prime}$, $r_{2}^{\prime}, d^{\prime}$, it computes the identity of the malicious spender by the following equations:

$$
\begin{aligned}
& \text { - } u=\frac{r_{1}-r_{1}^{\prime}}{r_{2}-r_{2}^{\prime}}\left(\bmod e_{B}\right), \\
& \text { - } \quad I D_{C}=g_{1}^{u}\left(\bmod n_{B}\right) .
\end{aligned}
$$

3.1.6 Exchange: In this phase, referring to the bank, the customer can exchange his old coin (which is not outdated) with new coins and update the expiration date of his own coin. To control the size of its database, this affair is undertaken by the following procedure:

Step 1. The customer:

(a) Offers his coin, besides his identity, to the bank.

\section{Step 2. The Bank B:}

(a) Checks deposit and exchange tables to ensure that the coin has nit already been exchanged or spent.

(b) Checks the authenticity of the customer and verification of the coin similar to the validation checking of the payment phase.

(c) Runs the withdrawal phase of the protocol.

(d) Updates the exchange table by inserting the information of the customer and the old coin.

\subsection{Weaknesses of Baseri, et al.,'s scheme}

In this subsection, we show some weaknesses of Baseri et al.'s scheme.

3.2.1 First Fault: Attacking Expiration Date: During time $t$ period, after successfully withdrawing a coin $\left(A^{\prime}, B, s_{1}, s_{2}, s_{3}, t\right)$, the spender can forgery a coin

$$
\left(A^{\prime *}, B^{*}, s_{1}^{*}, s_{2}^{*}, s_{3}^{*}, t^{\prime}\right)
$$

in time $t^{\prime}$ period. Here

$$
A^{\prime *}=A^{\prime}, B^{*}=B, s_{1}^{*}=s_{1}, s_{2}^{*}=s_{2}, s_{3}^{*}=s_{3}^{t / t^{\prime}} .
$$

Since

$$
s_{3}^{*}=s_{3}^{t / t^{\prime}}=\left(\left(A^{\prime}+B\right)^{1 / e_{B}^{* t}}\right)^{t / t^{\prime}}=\left(A^{\prime}+B\right)^{1 / e_{B}^{*} t^{\prime}}=\left(A^{\prime *}+B^{*}\right)^{1 / e_{B} * t^{\prime}} .
$$


So, $s_{1}^{*}, s_{2}^{*}$ and $s_{3}^{*}$ are the signatures of the bank on $A^{\prime *}, B^{*}$ and $A^{\prime *}+B^{*}$ in time $t^{\prime}$ period, which are signed with private keys $1 / e_{B}, 1 / e_{B}^{\prime}$ and $\left(1 /\left(e_{B} * t^{\prime}\right)\right)$, respectively. Furthermore, in payment, the spender computes $r_{1}^{*}$ and $r_{2}{ }^{*}$ using same number $s, x_{1}$ and $x_{2}$ in withdrawing the coin $\left(A^{\prime}, B, s_{1}, s_{2}, s_{3}, t\right)$. Let $d^{*}=H\left(A^{\prime *}, B^{*}, I D_{M}\right.$, date $\|$ time $)$. Obviously, $g_{1}^{r_{1}^{*}} g_{2}^{r_{2}^{*}}=A^{\prime * d^{*}} B^{*}$ holds. Hence, $\left(A^{\prime *}, B^{*}, s_{1}{ }^{*}, s_{2}{ }^{*}, s_{3}{ }^{*}, t^{\prime}\right)$ is valid coin in time $t^{\prime}$ period.

Note 1 This attack is only an expiration date attack. If the spender spends $\left(A^{\prime}, B, s_{1}, s_{2}, s_{3}, t\right)$ in time $t$ period, and also spends $\left(A^{\prime *}, B^{*}, s_{1}{ }^{*}, s_{2}{ }^{*}, s_{3}{ }^{*}, t^{\prime}\right)$ in time $t^{\prime}$ period, the bank can finds out the malicious spender. But if the spender only spends the coin $\left(A^{\prime *}, B^{*}, s_{1}{ }^{*}, s_{2}{ }^{*}, s_{3}{ }^{*}, t^{\prime}\right)$ in time $t^{\prime}$ period, the bank cannot finds out the malicious spender

3.2.2 Second Fault: Fault on Preventing Merchants Frauds: In practice, there are always many merchants from different shops. When merchant $M_{1}$ receives a coin $\left(A^{\prime}, B, s_{1}, s_{2}, s_{3}, t\right)$ from a spender who wants to buy goods from the merchant $M_{1}$, malicious merchant $M_{1}$ may send $\left(A^{\prime}, B, s_{1}, s_{2}, s_{3}, t\right)$ to a merchant $M_{2}$ to spend. When $M_{2}$ sends $d=H\left(A^{\prime}, B, I D_{M_{2}}\right.$,date $\|$ time $)$ to $M_{1}, M_{1}$ sends $d$ to the spender. After receiving $\left(r_{1}, r_{2}\right)$ from the spender, $M_{1}$ sends $\left(r_{1}, r_{2}\right)$ to $M_{2}$. Since $\left(r_{1}, r_{2}\right)$ satisfies $g_{1}^{r_{1}} g_{2}^{r_{2}}=A^{\prime d} B$. So, malicious merchant $M_{1}$ can spend the spender's coin $\left(A^{\prime}, B, s_{1}, s_{2}, s_{3}, t\right)$ to another merchant $M_{2}$. Due to lacking necessary authentication, in the above process, the spender cannot find any fraud. When the spender asks goods to $M_{1}, M_{1}$ can refuse him by saying something is wrong with the verification in payment phase. So, $M_{1}$ successfully carries out fraud. Baseri et al.'s scheme is not a practical scheme.

3.2.3 Third Fault: Fault on Anonymity: First we note that in Baseri, et al.,'s scheme coins have format $\left(A^{\prime}=A^{s}, B, s_{1}, s_{2}, s_{3}, t\right)$. Now we define coin $\left(A^{m}, B, s_{1}, s_{2}, s_{3}, t\right)$, $m \in Z_{n}$ as same roots coins with same $B$ determined by random numbers $x_{1}, x_{2} \in{ }_{R} Z_{e_{B}^{\prime}}^{*}$. When a spender send two same roots coins $\left(A^{m_{1}}, B, s_{1}, s_{2}, s_{3}, t\right)$ and $\left(A^{m_{2}}, B, s_{1}{ }^{*}, s_{2}, s_{3}{ }^{*}, t^{\prime}\right)$ to a merchant. Assumed $t^{\prime}>t$. The merchant may deliberately send

$$
d=d_{2}=d_{1}=H\left(A^{m_{1}}, B, I D_{M}, \text { date } \| \text { time }\right)
$$

to the spender. When the merchant receives $\left(r_{1}^{\prime}, r_{2}^{\prime}\right)$, he can compute the private key $u$ of the spender using $\left(r_{1}, r_{2}\right)$ related to $d_{1}$. Sine

$$
\begin{gathered}
r_{1}=m_{1} d u+x_{1}\left(\bmod e_{B}\right), r_{2}=m_{1} d+x_{2}\left(\bmod e_{B}\right) \\
r_{1}^{\prime}=m_{2} d u+x_{1}\left(\bmod e_{B}\right), r_{2}^{\prime}=m_{2} d+x_{2}\left(\bmod e_{B}\right) .
\end{gathered}
$$


The merchant can obtain the spender secret identity information $u=\frac{r_{1}-r_{1}^{\prime}}{r_{2}-r_{2}^{\prime}}\left(\bmod e_{B}\right)$. This violates the anonymity requirement of electronic cash.

Note 2 This attack is different from double spender detection, because

$$
\left(A^{m_{1}}, B, s_{1}, s_{2}, s_{3}, t\right) \neq\left(A^{m_{2}}, B, s_{1}{ }^{*}, s_{2}, s_{3}{ }^{*}, t^{\prime}\right)
$$

They are different coins. This attack indicates that among the random numbers, $x_{1}, x_{2} \in{ }_{R} Z_{e_{B}^{\prime}}^{*}$ and $s \in{ }_{R} Z_{n}^{*}$ in Baseri et al.'s scheme, $x_{1}, x_{2}$ are useful to protect the anonymity, but $s$ is almost no use. For security, spenders must choose different $x_{1}, x_{2}$ every time.

\section{The Proposed Scheme}

To overcome the weaknesses of Baseri, et al.,'s scheme, we proposed an improved electronic cash scheme. In our scheme there are also four participants: a Central Authority, the Bank, the Spender and the Merchant and the improved scheme contains five phases: initialization, withdrawal, payment, deposit and exchange. Initialization, deposit and exchange are as same as that of Baseri, et al.,'s scheme. Here we only describe the withdrawal and payment phases.

\section{Withdrawal phase}

To withdrawing and asking for a coin $\left(A^{\prime}, B, s_{1}, s_{2}, s_{3}\right)$ the following process is done:

Step 1. The spender S:

(a) Randomly Chooses $x_{1}, x_{2} \in{ }_{R} Z_{e_{B}^{\prime}}^{*}$ and $s, b_{1}, b_{2} \in{ }_{R} Z_{n}^{*}$

(b) Calculates: $A^{\prime}=A^{s}(\bmod n), B=g_{1}{ }^{x_{1}} g_{2}{ }^{x_{2}}(\bmod n)$,

$$
\omega_{1}=B^{t+s} b_{1}^{e_{B}^{\prime}}(\bmod n), \omega_{2}=\left(A^{\prime}+B^{t+s}\right) b_{2}^{\left(e_{B} *^{*}\right)}(\bmod n) .
$$

(c) Sends $t, \omega_{1}, \omega_{2}$ to the bank.

Step 2. The bank B:

(a) Checks the validity of the Date/Time.

(b) Signs $\omega_{1}, \omega_{2}$ by generating:

$$
O_{2}=\omega_{1}^{1 / e_{B}^{\prime}}(\bmod n), O_{3}=\omega_{2}^{1 /\left(e_{B} * t\right)}(\bmod n) .
$$

(c) Sends $\mathrm{O}_{2}$ and $\mathrm{O}_{3}$ to the spender.

Step 3. The spender S:

(a) Verifies the following equations

$$
O_{2}^{e_{B}^{\prime}}=\omega_{1}(\bmod n), O_{3}^{e_{B}{ }^{* t}}=\omega_{2}(\bmod n)
$$

(b) Gains the three signatures $s_{1}, s_{2}, s_{3}$ of the bank on $A^{\prime}, B^{t+s}$ and $A^{\prime}+B^{t+s}$, respectively.

$$
s_{1}=O_{1}^{s}(\bmod n), s_{2}=O_{2} / b_{1}(\bmod n), s_{3}=O_{3} / b_{2}(\bmod n) .
$$

The Coin is $\left(A^{\prime}, B^{t+s}, s_{1}, s_{2}, s_{3}, t\right)$.

\section{Payment phase}

To spend a coin at the shop, the following steps are done Step 1. The spender S:

(a) Sends $\left(A^{\prime}, B^{t+s}, s_{1}, s_{2}, s_{3}, t\right)$ to the merchant $\mathrm{M}$. 
Step 2. The merchant M:

(a) Checks the expiration date of the coin.

(b) Verifies the three signatures, $s_{1}, s_{2}, s_{3}$ of the bank on $A^{\prime}, B^{t+s}$ and $A^{\prime}+B^{t+s}$, respectively, via the following formulas

$$
s_{1}^{e_{B}}=A^{\prime}, \quad s_{2}^{e_{B}^{\prime}}=B^{t+s}, s_{3}^{e_{B} \cdot t}=A^{\prime}+B^{t+s}
$$

(c) Computes $d=H\left(A^{\prime}, B^{t+s}, I D_{M} \text {, date } \| \text { time }\right)^{1 / e_{M}}$, Here $H$ is the hash function determined in the initialization phase, $e_{M}$ is the public key of the merchant, $I D_{M}$ is the identity of the merchant and datell time represents the date and time of the transaction.

(d) Sends $d$ and datell time to the spender.

Step 3. The spender S:

(a) Verifies the signature $d$ of the merchant, using the public key $e_{M}$ of the merchant $M$ via the following formulas

$$
d^{e_{M}}=H\left(A^{\prime}, B^{t+s}, I D_{M} \text {, date } \| \text { time }\right)
$$

(b) Computes

$$
r_{1}=d u s+(t+s) x_{1}\left(\bmod e_{B}\right), r_{2}=d s+(t+s) x_{2}\left(\bmod e_{B}\right) .
$$

(c) Sends $r_{1}$ and $r_{2}$ to the merchant.

Step 4. The merchant $\mathrm{M}$ accepts the coin if and only if $g_{1}^{r_{1}} g_{2}^{r_{2}}=A^{\prime d} B^{t+s}$.

\section{Security Analysis}

\subsection{Immunity to the Proposed Attacks}

The improved scheme is not subjected to the proposed attacks on Baseri, et al.,'s scheme.

Firstly, to avoid expiration date attack, we set $\omega_{2}=\left(A^{\prime}+B^{t+s}\right) b_{2}^{\left(e_{B}{ }^{*} t\right)}(\bmod n)$ and $s_{3}=O_{3} / b_{2}(\bmod n)$. So, $s_{3}$ is the sign of the bank on $A^{\prime}+B^{t+s}$. Now, if the spender computes $s_{3}{ }^{t / t^{\prime}}$, he can get $\left(A^{\prime}+B^{t+s}\right)^{1 / e_{B}^{* t^{\prime}}}$. But, he cannot get $\left(A^{\prime}+B^{t^{\prime}+s}\right)^{1 / e_{B} * t^{\prime}}$. So, the improved scheme is not subjected to expiration date attack.

Secondly, in improved scheme, when the spender sends $\left(A^{\prime}, B^{t+s}, s_{1}, s_{2}, s_{3}, t\right)$ to the merchant, the merchant computes $d=H\left(A^{\prime}, B^{t+s}, I D_{M} \text {, date } \| \text { time }\right)^{1 / e_{M}}$, not computing $d=H\left(A^{\prime}, B^{t+s}, I D_{M}\right.$, datell time $)$. When the merchant sends $d$ to the spender, the spender first verifies the signature $d$ of the merchant, using the public key $e_{M}$ of the merchant $M$. If $d$ does not satisfy the verification equation, the spender does not send $r_{1}$ and $r_{2}$ to the merchant. So, the improved is not subjected to merchants fraud attack.

Thirdly, in the improved scheme the coin $\left(A^{\prime}, B^{t+s}, s_{1}, s_{2}, s_{3}, t\right)$ is different from the coin $\left(A^{\prime}, B, s_{1}, s_{2}, s_{3}, t\right)$ in Baseri, et al.,'s scheme. $B^{t+s}$ is not only related to random numbers $x_{1}, x_{2}$, but also related to time $t$ and random number $s$. So, the number of same root coins can be largely reduced in improved scheme. The anonymity of electronic cash in new scheme can be efficiently protected. 


\subsection{Anonymity}

In the first place, while obtaining the signatures $s_{2}, s_{3}$ of the bank on $B^{t+s}$, $A^{\prime}+B^{t+s}$ respectively, the spender blind $B^{t+s}$ and $A^{\prime}+B^{t+s}$ by blinding factors. So, the attacker cannot get spender's identity information in the withdrawal phase. Furthermore, no one can know the identity of the spender by the information of payment phase. The information in the payment phase of the scheme includes the coin $\left(A^{\prime}, B^{t+s}, s_{1}, s_{2}, s_{3}, t\right)$ and $\left(r_{1}, r_{2}\right)$. Although $A^{\prime}=A^{s}$, due to the difficulty in computing discrete logarithm, the attacker cannot get $s$ from $A^{\prime}$, and in the equations to compute $r_{1}, r_{2}$, there are $r_{1}, r_{2}, x_{1}, x_{2}, s$, five unknown numbers and time parameter $t$. It reveals no information to the attacker.

\subsection{Double Spender Detection}

In the case that a spender spends a coin twice or more, the identity information of the malicious spender can be obtained from the equations:

$$
u=\frac{r_{1}-r_{1}^{\prime}}{r_{2}-r_{2}^{\prime}}, I D_{C}=g_{1}^{u}\left(\bmod n_{B}\right)
$$

Here $\left(r_{1}, r_{2}\right)$ and $\left(r_{1}^{\prime}, r_{2}^{\prime}\right)$ are information the spender sends to the merchant in payment phase in twice consumption, respectively. In fact,

$$
\begin{gathered}
r_{1}=d u s+(t+s) x_{1}\left(\bmod e_{B}\right) \\
r_{1}^{\prime}=d^{\prime} u s+(t+s) x_{1}\left(\bmod e_{B}\right) \\
r_{2}=d s+(t+s) x_{2}\left(\bmod e_{B}\right) \\
r_{2}^{\prime}=d^{\prime} s+(t+s) x_{2}\left(\bmod e_{B}\right)
\end{gathered}
$$

So,

$$
u=\frac{r_{1}-r_{1}^{\prime}}{r_{2}-r_{2}^{\prime}}
$$

Further, one can obtain the identify information of the malicious spender by

$$
I D_{C}=g_{1}^{u}\left(\bmod n_{B}\right)
$$

\subsection{Unforgeability}

If an adversary intends to forge a coin $\left(A^{\prime *},\left(B^{t+s}\right)^{*}, s_{1}{ }^{*}, s_{2}{ }^{*}, s_{3}{ }^{*}, t^{*}\right)$, he must generate three signatures $s_{1}{ }^{*}, s_{2}{ }^{*}, s_{3}{ }^{*}$ for $A^{\prime *},\left(B^{t+s}\right)^{*},\left(A^{\prime}+B^{t+s}\right)^{*}$, respectively. The adversary may get the two signatures $s_{1}{ }^{*}, s_{2}{ }^{*}$ for $A^{\prime *},\left(B^{t+s}\right)^{*}$, respectively. But, he cannot get the signature $s_{3}{ }^{*}$ for $\left(A^{\prime}+B^{t+s}\right)^{*}$.

After get a cion $\left(A^{\prime}, B^{t+s}, s_{1}, s_{2}, s_{3}, t\right)$, the adversary can choose a random number $a \in{ }_{R} Z_{n}^{*}$, and let

$$
A^{\prime *}=A^{\prime a},\left(B^{t+s}\right)^{*}=\left(B^{t+s}\right)^{a}, s_{1}^{*}=s_{1}{ }^{a}, s_{2}{ }^{*}=s_{2}{ }^{a} .
$$

Now, $s_{1}{ }^{*}, s_{2}{ }^{*}$ are signatures for $A^{\prime *},\left(B^{t+s}\right)^{*}$, respectively. But the adversary cannot obtain the signature $s_{3}{ }^{*}$ for $\left(A^{\prime}+B^{t+s}\right)^{*}$. 
On the other hand, if the adversary lets $s_{3}{ }^{*}=s_{3},\left(A^{\prime}+B^{t+s}\right)^{*}=A^{\prime}+B^{t+s}$, and divides $\left(A^{\prime}+B^{t+s}\right)^{*}=A^{\prime}+B^{t+s}=A^{\prime *}+\left(B^{t+s}\right)^{*}, A^{\prime *} \neq A^{\prime},\left(B^{t+s}\right)^{*} \neq B^{t+s}$ Due to the hardness of discrete logarithm problem, the adversary cannot compute the signatures $s_{1}{ }^{*}, s_{2}{ }^{*}$ for $A^{\prime *},\left(B^{t+s}\right)^{*}$, respectively. So, the adversary cannot generate new coin by forgery.

\section{Performance Comparison}

Baseri, et al., compared their scheme with some other related scheme $[2,12,16]$. The comparison showed that Baseri, et al.,'s scheme cost less computation time. But we show Baseri, et al.,'s scheme is subjected to some weaknesses. For developing immunity from attacks, we propose a new scheme. Here we just compare our scheme with Baseri, et al.,'s scheme. Compared with Baseri, et al.,'s scheme, the new scheme just increases two modular multiplications in withdrawal phase and payment phase, respectively. But our scheme is more secure. So, from security and efficiency, our scheme needs less computation and communication costs.

\section{Conclusion}

Electronic payment is one of the key issues of electronic commerce development. Electronic cash is special electronic payment. There are a number of requirements for secure electronic cash schemes, such as, anonymity, unforgeability, unreusability, date attachability, divisibility, transferability and portability. In this paper, we show Baseri, et al.,'s electronic cash scheme is suffering from some weaknesses in anonymity, expiration date and merchant frauds. To improve Baseri, et al.,'s scheme, we propose a new off-line electronic cash scheme. We also discuss the security properties of our scheme, such as, anonymity, double spender detection and unforgeability. It is worthy to be mentioned that the new scheme not only possesses the features, such as anonymity, unforgeability, unreusability, but also possesses the feature of avoiding merchant frauds.

\section{Acknowledgements}

This work was supported by the Research Programs of Applied Basic and Advanced Technology of Tianjin (No. 15JCYBJC15900).

\section{References}

[1] D. Chaum, "Blind signatures for untraceable payments", In Crypto 82, Plenum Press, New York, (1983), pp. 199-203.

[2] Z. Eslami and M. Talebi, "A new untraceable off-line electronic cash system", Electronic Commerce Research and Application, vol. 10, (2011), pp. 59-66.

[3] R. Anderson, C. Manifavas and C. Sutherland, "NetCar-A practical electronic cash system", In Security Protocols, Springer, (1997), pp. 49-57.

[4] G. Davida, Y. Frankel, Y. Tsiounis and M. Yung, "Anonymity control in e-cash systems", In Financial Cryptography, Springer, (1997), pp. 1-16.

[5] G. Maitland and C. Boyd, "Fair electronic cash based on a group signature scheme", Information and Communication Security, (2001), pp. 461-465.

[6] D. Chaum and S. Brands, "Minting electronic cash", Spectrum, IEEE, vol. 34, no. 2, (2002), pp. 30-34.

[7] J. Camenisch, S. Hohenberger and A. Lysyanskaya, "Compact e-cash", Advances in Cryptology, EUROCRYPT, (2005), pp. 302-321.

[8] H. Wang and Y. Zhang, "Untraceable off-line electronic cash flow in e-commerce", $24^{\text {th }}$ Australasian Computer Science Conference Proceedings. IEEE, (2002), pp. 191-198.

[9] S. Brands, "Untraceable off-line cash in wallet with observers", In Advances in CryptologyCRYPTO’93, Springer, (1994), pp. 302-318. 
[10] C. Ku, C. Tsao, Y. Lin and C. Chen, "An escrow electronic cash system with limited traceability", Information Science, vol. 164, no. 1-4, (2004), pp. 17-30.

[11] T. Cao, D. Lin and R. Xue, "A randomized RSAbased partially blind signature scheme for electronic cash", Computer \&Security, vol. 24, no. 1, (2005), pp. 44-49.

[12] W. Juang, "D-cash: a flexible pre-paid e-cash scheme for date-attachment", Electronic Commerce Research and Applications, vol. 6, no. 1, (2007), pp. 74-80.

[13] C. Fan and W. Sun, "Efficient encoding scheme for date attachable electronic cash", The $24^{\text {th }}$ Workshop on Combinatorial Mathematics and Computation Theory, (2007), pp. 405-410.

[14] Y. Baseri, B. Takhtaei and J. Mohajeri, "Secure untraceable off-line electronic cash system", Scientia Iranica, vol. 20, no. 3, (2013), pp. 637-646.

[15] R. Rivest, A. Shamir and L. Adleman, "A method for obtaining digital signature and public-key cryptosystems", Communications of the ACM, vol. 21, no. 2, (1978), pp. 120-126.

[16] R. Martinez-Pelaez, F. Rico-Novella, and C. Satizabal, "Tomin: trustworthy mobile cash with expiration-date attached". Journal of Software, vol. 5, no. 6, (2010), pp. 579-584.

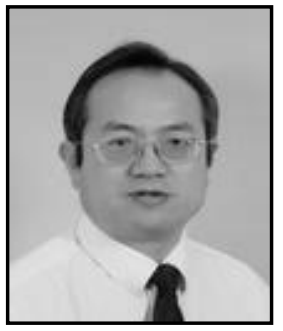

Baoyuan Kang, received M.S. in algebra from the shanxi University, and ph.D. in cryptography from Xidian University, People's Republic of China in 1993 and 1999, respectively. From 1993 to 1999, he taught mathematics in Northwestern Polytechnic University. Since 1999 he has taught mathematics and computer science in Central South University. Now he is a professor at Tianjin Polytechnic University. His current research interests are cryptography and information security.

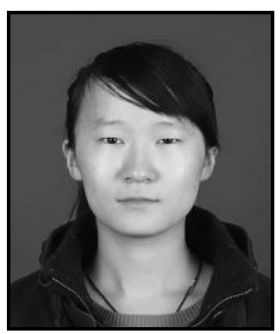

Danhui Xu, received B.S. in Computer Science from the Tianjin Polytechnic University, China in 2013. Now he is a postgraduate student at Tianjin Polytechnic University. His current research interests are cryptography and information security. 
International Journal of Hybrid Information Technology

Vol.9, No.1 (2016) 